\title{
A compact high-resolution spectrometer based on a segmented conical crystal analyzer
}

Cite as: Rev. Sci. Instrum. 91, 043105 (2020); https://doi.org/10.1063/1.5141758

Submitted: 06 December 2019 . Accepted: 02 April 2020 . Published Online: 20 April 2020

José I. Robledo (D), Carlos A. Pérez (D), and Héctor J. Sánchez

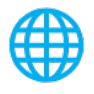

\section{Lock-in Amplifiers up to $600 \mathrm{MHz}$}
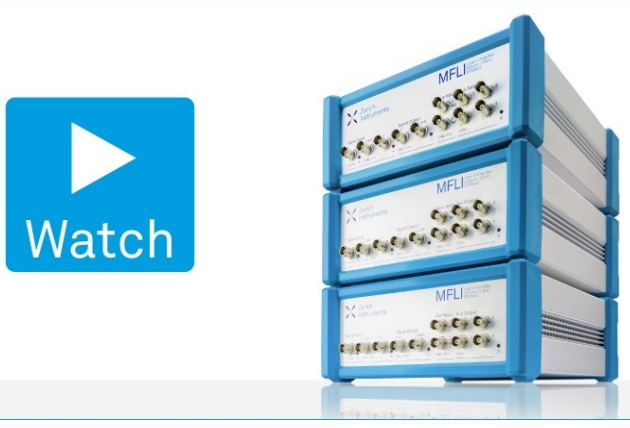


\title{
A compact high-resolution spectrometer based on a segmented conical crystal analyzer
}

\author{
Cite as: Rev. Sci. Instrum. 91, 043105 (2020); doi: 10.1063/1.5141758 \\ Submitted: 6 December 2019 - Accepted: 2 April 2020 • \\ Published Online: 20 April 2020

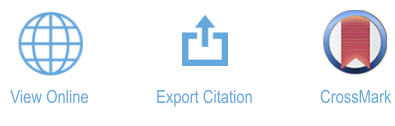

José I. Robledo, ${ }^{1,2, a)}$ (D) Carlos A. Pérez, ${ }^{3}$ (D) and Héctor J. Sánchez ${ }^{2,4}$

\begin{abstract}
AFFILIATIONS
${ }^{1}$ Centro Atómico Bariloche (CAB-CNEA), San Carlos de Bariloche 8400, Argentina

${ }^{2}$ National Scientific and Technical Research Council (CONICET), Córdoba 5000, Argentina

${ }^{3}$ Brazilian Synchrotron National Laboratory (LNLS), Brazilian Center for Research in Energy and Materials (CNPEM), Campinas-SP 13084-970, Brazil

${ }^{4}$ Facultad de Matemática, Astronomía y Física, Universidad Nacional de Córdoba (UNC), Córdoba 5000, Argentina
\end{abstract}

a) Author to whom correspondence should be addressed: jorobledo@unc.edu.ar

\begin{abstract}
In this work, the design, fabrication, and evaluation of a compact, one-shot spectrometer based on a segmented conically bent crystal analyzer are described. The system is a "one-shot" wavelength dispersive spectrometer, which has a crystal analyzer with an innovative geometry. It reaches an energy resolution of around $8 \mathrm{eV}$ for $\mathrm{Mn} \mathrm{K} \alpha_{1}$ line, which is at least an order of magnitude better than the commonly used energy dispersive spectrometers for fluorescence, and is comparable to current wavelength dispersive spectrometers. The prototype spectrometer fabricated in this work avoids angle scans that most wavelength dispersive spectrometers require, has the advantage of a sample-detector distance of only $146 \mathrm{~mm}$, and allows for the simultaneous measurement of approximately a $2 \mathrm{keV}$ window. This system is suitable to be used at synchrotron radiation facilities and free electron lasers, and it can even be adapted to an x-ray tube in any conventional x-ray laboratory.
\end{abstract}

Published under license by AIP Publishing. https://doi.org/10.1063/1.5141758

\section{INTRODUCTION}

Practical X-ray spectroscopy has been characterized by the resolution of its different stages (excitation/detection). Historically, the excitation channel was characterized by low energy resolution photons emitted by $\mathrm{x}$-ray tubes. Secondary targets and radioactive sources represented a limited alternative. With the advent of nonconventional sources, such as storage rings, and the possibility of using crystal monochromators, high-resolution excitation became a reality and even gave places to techniques, such as Extended X-ray Absorption Fine Structure (EXAFS), X-ray Absorption Near-Edge Structure (XANES), and Energy Dispersive Inelastic X-ray Scattering (EDIXS), among others. On the detection channel, the situation was led by two main approaches: the so-called Wavelength Dispersive Spectrometers (WDSs) and the Energy Dispersive Spectrometers (EDSs). WDS systems are based on Bragg's law and consist of a moving secondary crystal that performs an angular scan. In this way, photons of different energies are collected by means of a proportional counter. They are characterized by a high resolution (i.e., a few eV) but are much time consuming and normally present a complex arrangement that complicates placing them in reduced spaces. On the other hand, EDSs are based on solid state detectors that can perform a fast collection of a high flux of photons on a simple experimental setup. The price is a low-resolution energy (typically about $100 \mathrm{eV}$ ). This has been the dilemma in $\mathrm{x}$-ray spectrometry for years: high resolution in complicated and slow systems vs poorer resolution detectors in simpler and faster setups. Nevertheless, the requirements of modern science are far beyond dichotomies. Today, the paradigm of time-resolved analyses with high resolution is a must, and new innovative instruments are needed.

The efficiency of spectroscopic techniques normally depends on the energy resolution of the detector used. Techniques such as X-ray Fluorescence (XRF), ${ }^{1}$ X-ray Emission Spectroscopy (XES), ${ }^{2}$ $\mathrm{X}$-ray Absorption Spectroscopy in fluorescent mode (FY-XAS), ${ }^{3}$ and even Resonant Inelastic X-ray Scattering (IXS, EDIXS) ${ }^{4}$ require 
a detection system with the highest achievable energy resolution. The upcoming fourth-generation synchrotron radiation source SIRIUS in Campinas, Brazil, has pushed forward the in-house development of new detector systems for improving counting capabilities and readout electronics. Furthermore, the significant increase in photon flux delivered by this high-brilliance source opens up unique opportunities for the user community to perform more efficient experiments in x-ray spectroscopy that are naturally "photon-hungry" when crystal analyzers are used as to improve the energy resolution.

$\mathrm{X}$-ray fluorescence is a well-established technique that has been of great interest since the mid-twentieth century. It has been exploited in many different applications, such as art, geology, chemistry, and physics, among others. Particularly, it has been used in earth sciences, with geological purposes, in the attempt of quantifying elemental concentrations in bulk samples. Nevertheless, the lack of enough resolution for a broad energy range in the L-lines of lanthanides has always been a problem. Many works have tried to overcome this by applying multivariate statistical analyses as to separate the information given in the energy region of interest (ROI) in different parts, corresponding to the different spectral lines present. ${ }^{5,6}$ Nevertheless, it would be useful to have a spectrometer capable of resolving the L emission lines successfully. Reviewing the emission lines of these elements, an energy resolution of around $10 \mathrm{eV}$ is enough to allow for the desired discrimination.

In this work, we propose a modern wavelength dispersive spectrometer scheme. The concept of a segmented conical crystal analyzer is described, and a compact spectrometer based on such a crystal is used to measure XRF spectra with few $\mathrm{eV}$ of resolution in a wide energy region and in a single exposure at the XRF-D09B beamline of the Brazilian synchrotron light source (LNLS).

There are many detection systems based on a WDS approach that are in use nowadays. ${ }^{7-10}$ The differences in reachable energies and the dimensions of the system are determined mainly by the crystal choice and its geometry. A nice example is a system developed by Szlachetko et al. ${ }^{11}$ in which a crystal analyzer with cylindrical shape was proposed and tested. In their work, they obtained an energy resolution of around $1 \mathrm{eV}$ for the $\mathrm{Mn}$ $\mathrm{K} \alpha$ line. Nevertheless, as mentioned before, there is a compromise between the energy resolution capability and the spectrometer's dimension. In this example, the distance between the sample and the detector was approximately $250 \mathrm{~mm}$. In these dimensions, attenuation in the air is clearly a problem for energies around a few $\mathrm{keV}$ (a $5 \mathrm{keV}$ photon would be completely attenuated). Therefore, this compromise is a point to be considered when designing a WDS. Moreover, synchrotron beamlines and conventional laboratories normally do not have much space to place new spectrometers if the device was not planned before the commissioning of the sample environment. Compact systems are very convenient in this way.

Morishita et al. ${ }^{12}$ proposed an integrated conical crystal analyzer in which they achieved the desired crystal shape by pyroclastic deformation. ${ }^{13}$ They proposed an approach on the surface needed by performing a spline integration of the surface and by pressing the crystal with an upper and lower die having the integrated surface shape. The problem arises after bending the crystal by pyroclastic deformation since strains affect the lattice plane distances.
This introduces a loss in energy resolution due to the inhomogeneity of the lattice constant, which is described in their work. It is also a difficult process to achieve successfully, and many crystal wafers may be used until the desired shape is obtained.

In order to overcome these difficulties, we propose in this work to generate the crystal's surface by defining discretely the corresponding conical rings to achieve focalization in the perpendicular plane, as proposed by Morishita et al. In a good approximation, it is possible to append many consecutive conical rings of a determined width and to achieve a smooth surface that fulfills Bragg's condition for photons in each point in such a way as to allow for energy focalization and discrimination. In this way, focalization is better achieved on the vertical plane as there is no interpolation procedure. In order to acquire a spectrum in one shot, a position-sensitive detector (PSD) is needed, since, due to the crystal's surface geometry, photons with different energies will focalize in different positions on a convenient axis, reducing the sample-todetector distance (when compared to cylindrical or planar geometries). Another optimization that we show in this work is the dicing of the crystal in small rhombohedral pieces. This allows the crystal to reproduce the surface shape without much stress on the lattice planes.

\section{EQUATIONS}

The experimental setup and the basic parameters are sketched in Fig. 1 in which the three main parts of the spectrometer are shown: the sample, the discrete conical crystal analyzer, and the position sensitive detector. The crystal analyzer's shape that we propose consists of several conical sections of width $w$ that vary their radii as a function of the position. As to understand this construction, two conical segments are shown in Fig. 1 with different colors. The first one is shown at the beginning of the crystal analyzer and has a radius $R_{0}$. The second one is shown at the end of the crystal and has a radius $R_{i}$ and a tilt angle $\delta_{i}$. Since these conical sections are at different distances from the emitting source, their corresponding Bragg angles differ; therefore, they diffract different photon energies.

Taking this into account, the parameters that determine the crystal's geometry are (1) the distance between the source and the crystal's edge $\left(L_{0}\right),(2)$ the crystal's curvature at its edge $\left(R_{0}\right)$, and (3) the crystal's interplanar distance (d) (Fig. 1).

We propose the following discrete equations depending on the parameter $w(=1 \mathrm{~mm}$, in our case) for the parameterization of the crystal analyzer's surface: ${ }^{14}$

$$
\begin{gathered}
x_{i}=x_{i-1}+w, \\
\theta_{i}=\tan ^{-1}\left(\frac{R_{0}}{x_{i}+L_{0}}\right), \\
\delta_{i}=\tan ^{-1}\left(\frac{\left(R_{0}-\left(2 L_{0}-x_{i}\right)\right) \tan \theta_{i}}{2 L_{0}}\right), \\
R_{i}=R_{0} \frac{\sin \left(\theta_{i}-\delta_{i}\right)}{\sin \theta_{i}},
\end{gathered}
$$




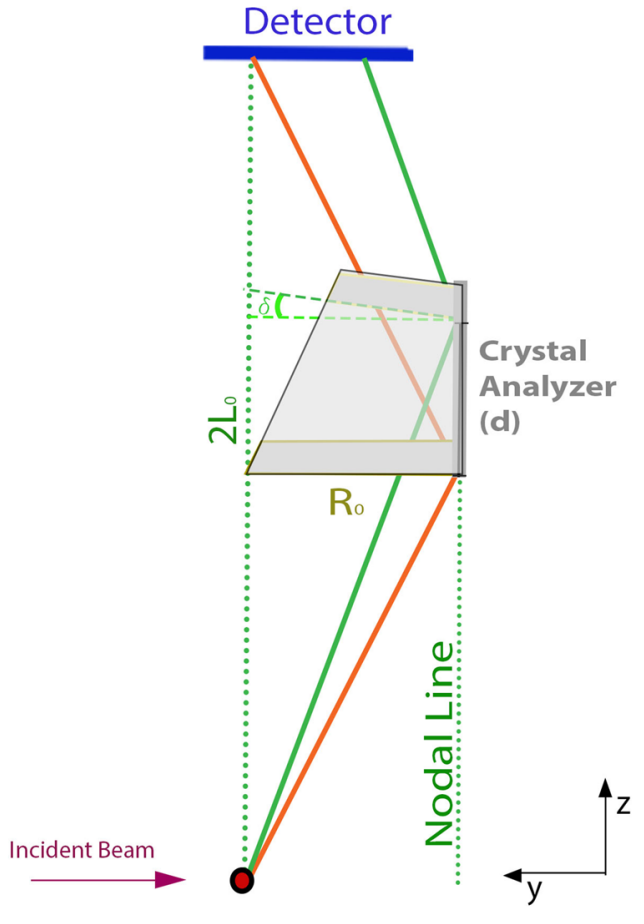

FIG. 1. Schematic of the design of the crystal analyzer.

where $x_{i}$ is the position of the $i$ th section, $R_{0}$ and $2 L_{0}$ are the radii of the first section and the distance between the sample and the detector, respectively, and $\theta_{i}, R_{i}$, and $\delta_{i}$ are the angle for which Bragg's law is fulfilled, the curvature radius, and the inclination angle of the $i$ th conical section, respectively. With these equations, it is necessary to fix $x_{0}=L_{0}$, and an iterative process may be performed to obtain the values for each of the following conic sections. The constraint for $x_{0}$ means that the crystal must be located halfway between the sample and the detector. The energy that each of these conical section diffracts can be obtained by

$$
E_{i}(\mathrm{keV})=\frac{12.398}{2 d_{h k l}(\AA) \sin \theta_{i}},
$$

where $d_{h k l}$ is the interplanar distance of the crystal used, with Miller indices $(h, k, l)$.

\section{DESIGN PARAMETERS}

As to test the proposed equations, a crystal prototype had to be fabricated. These equations do not have a unique solution and mostly depend on the crystal selection, how it is cut $\left(d_{h k l}\right)$, and on the desired energy range to be used in a one-shot measurement. Hence, our first concern was that the crystal selection and its geometry should be able to discriminate photons in the desired energy region. From the crystal selection point, we had available a $\mathrm{Si}(220)$ crystal wafer for testing. Concerning the energy region, one of our goals was to be able to discriminate the main $\mathrm{L}$ emission lines from different rare earth elements. These emission lines lie mostly between $5 \mathrm{keV}$ and $7 \mathrm{keV}$. Some K-lines of typical transition metal standards, such as $\mathrm{Mn}$ and $\mathrm{Cr}$, also lie in this energy range.

First, attenuation in air should be reduced in order to keep good counting statistics at the collecting $\mathrm{x}$-ray sensor; therefore, the maximum possible distance between the sample and this sensor was limited to $150 \mathrm{~mm}\left(2 L_{0}\right)$. This was calculated taking into account the air absorption coefficient and the maximum length, and a 5 $\mathrm{keV}$ photon would travel in the proposed configuration. This selection also guaranteed the compactness of the spectrometer. Since the crystal must be located at $L_{0}$ from the sample, the limiting maximum length of the crystal is $75 \mathrm{~mm}$. Therefore, Eqs. (1)-(5) were solved iteratively $(i=1, \ldots, 75)$ using a code written in MATLAB ${ }^{15}$ for different combinations of parameters $L_{0}$ and $R_{0}$. A list of values of the variables $\theta_{i}, \delta_{i}, R_{i}, x_{i}$ and the corresponding energies $E_{i}$ was obtained (an example of this list is given in the supplementary material). For each of these combinations, a different number of conical sections were needed to achieve the desired energy range. A few combinations of parameters fulfilled all our needs. Therefore, as to select one, the geometry of the crystal was tested by simulating the interaction of random photons with the crystal for each corresponding experimental configuration. The simulations were carried out with McXtrace. ${ }^{16}$ This open-source software allows performing $\mathrm{x}$-ray tracing of photons, which are generated with a certain degree of randomness through the Monte Carlo method. It is specially intended for synchrotron beamline component testing. The software allows creating specific components in which the photons may interact. Component creation can be done by programming in $\mathrm{C}$ language. We made a specific surface by creating a conically bent section of width $w=1 \mathrm{~mm}$ and repeating this surface $n$ times for different cone proportions (i.e., different $\delta$ ), where $n$ is the number of sections that allowed to study the entire energy range. Since photons are discriminated by their Bragg angle, this type of spectrometer requires a Position Sensitive Detector (PSD), which is shown in Fig. 3. A PSD is included in McXtrace as an internal predefined component.

As for the simulations, a description of the emitting source (sample), the crystal analyzer, and the PSD must be given, with their respective spatial positions. A "perfect" emitting manganese sample was described by setting the emission energies of the source and the corresponding emission probabilities, as in Table I. ${ }^{17}$ It may be seen that the difference between $K \alpha$ lines is around $10 \mathrm{eV}$.

The results at the PSD after simulating the emission of $5 \times 10^{9}$ photons focalized at the crystal's surface for the best parameter configuration are shown in Fig. 2. The choice of the number of photons was made to simulate the conditions under which the crystal would be tested, i.e., the incoming photon-flux available at the D09B-XRF beamline. Clearly, the photons are diffracted by the crystal's surface and focalized at different positions of the PSD.

TABLE I. K-emission line energies for electron K-shell transitions of manganese, used as a photon source.

\begin{tabular}{lccc}
\hline \hline Emission line & Transition & Energy $(\mathrm{eV})$ & Emission rate \\
\hline$K \alpha_{2}$ & $K-L_{I I}$ & 5887.6 & 0.29776 \\
$K \alpha_{1}$ & $K-L_{I I I}$ & 5897.0 & 0.58416 \\
$K \beta$ & $K-M_{I I-I I I}$ & 6490.4 & 0.11808 \\
\hline
\end{tabular}




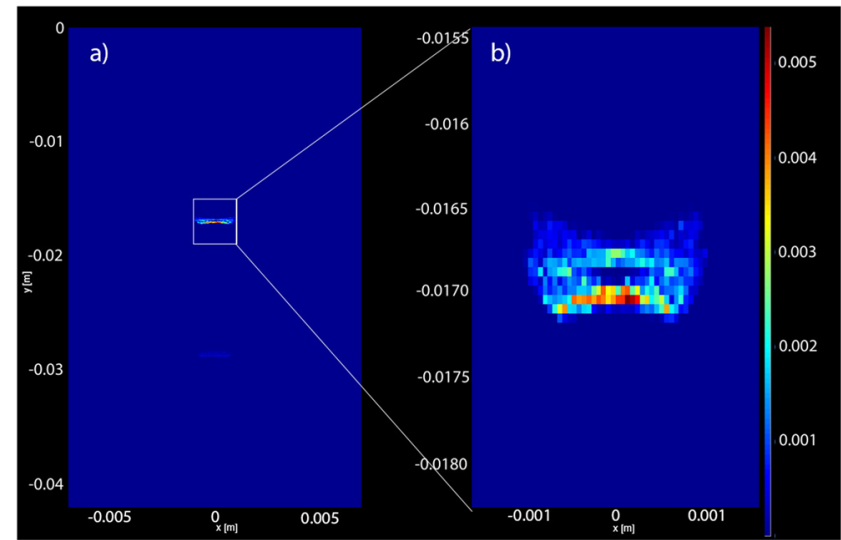

FIG. 2. Two-dimensional imaging of Mn K-emission lines reaching the PSD after simulating the interaction with the crystal analyzer. In (a), $K \alpha$ and $K \beta$ lines may be appreciated, and in (b), a zoomed-in view of the $K \alpha$ region allows us to observe the fine structure.

The only variable of interest is the $y$-axis since the twodimensional images will be integrated through the $\mathrm{x}$-axis to obtain the final XRF spectrum. The resulting calibrated and normalized spectrum is shown in Fig. 3 in which only the $K \alpha$ region is shown. This spectrum has been calibrated in energy by the following equation:

$$
E_{i}(\mathrm{keV})=\frac{12.398}{2 d \sin \left(\tan ^{-1}\left(\frac{R_{0}}{L_{0}\left(1+\frac{(i-1) s_{p x}}{2 R_{0}-(i-1) s_{p x}}\right)}\right)\right)},
$$

where $L_{0}, R_{0}$, and $d$ are the input parameters described in Sec. II and $s_{p x}$ is the length in the y-axis direction of the PSD pixels

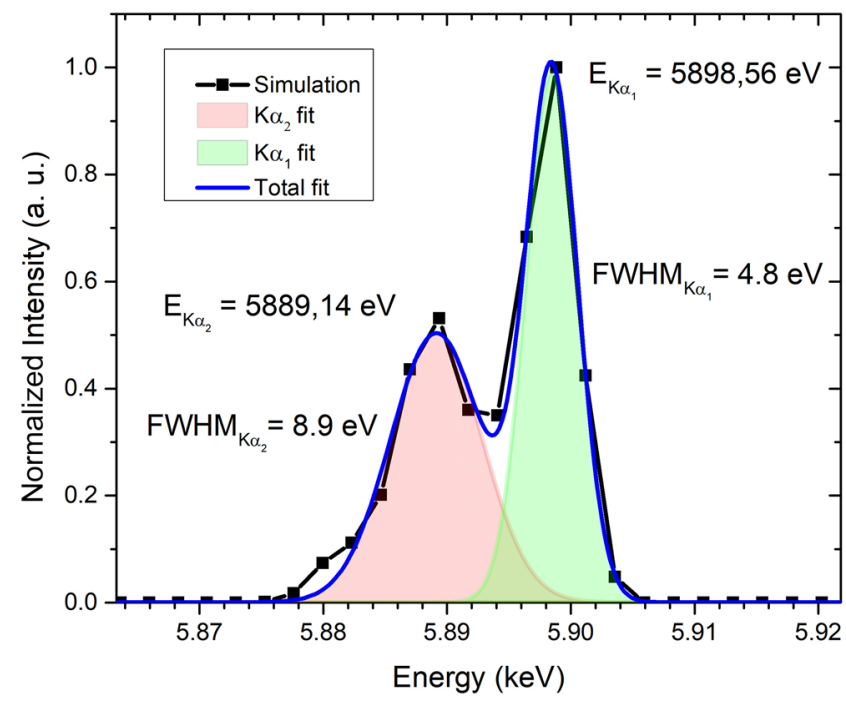

FIG. 3. Resulting $K \alpha$ energy region of the spectrum obtained by the Monte Carlo simulation of a Mn source (black points) and the corresponding fit (blue line).
(0.056 $\mathrm{mm}$ in our case). As it may be seen from Eq. (6), the energy resolution depends on the pixel size. As the pixel size becomes smaller, the resolution in energy is improved. It is important to mention that a Darwin width parameter was taken into account for the acceptance of the Bragg law fulfillment in the crystal interaction simulation. Since the available crystal was a $\mathrm{Si}(220)$, its Darwin width $\left(\omega_{s}=9.0687\right.$ arc sec $)$ was used, from which a $\delta \theta_{5.3 \mathrm{keV}}$ $=(37.4927 \pm 0.0025)^{\circ}$ was considered as the angular acceptance for the Bragg law. It is also important to note that the design of this spectrometer is based on a point-like source; therefore, aberrations will be present in the PSD array due to finite-size sources. For the simulations, a finite-size source was used with a size corresponding to the smallest achievable focal spot in the sample available at the D09B-XRF beamline by means of a KB-mirror system.

The fitting of the $K \alpha_{1}$ and $K \alpha_{2}$ peaks is also shown in Fig. 3. Gaussian shapes were used for the fitting since they correspond to fluorescence peaks, and the main estimated parameters are shown, which can be compared to those in Table I.

This simulation resulted in an energy resolution of around 5-9 eV for the $M n-K \alpha_{1}$ line. The final crystal parameters were $R_{0}=56 \mathrm{~mm}, L_{0}=73 \mathrm{~mm}, d=1.92 \AA$, and $w=1 \mathrm{~mm}$. With these parameters, thirty-six $1 \mathrm{~mm}$ conical slices attached can analyze the energy region from $5.3 \mathrm{keV}$ to $7 \mathrm{keV}$ if a $40 \mathrm{~mm}$ long PSD is available. Note that the total length of the crystal will result in $36 \mathrm{~mm}$, which is nearly half the length of $L_{0}$ between the starting point of the crystal and the PSD.

Once these parameters have been defined, it is possible to calculate some figure of merit in order to compare this spectrometer with other available WDS. An interesting one is the total solid angle $(\Omega)$ covered by a segmented-type crystal. This quantity may be approximated by the ratio of the crystal area perpendicular to the incident radiation to the squared distance between the crystal and the source. ${ }^{10}$ In a cylindrical geometry, this results in

$$
\Omega=N_{s} \frac{S_{x} S_{y}}{R^{2}} \sin ^{3} \theta
$$

where $N_{s}$ is the number of crystal segments and $S_{x}$ and $S_{y}$ are the segment width and segment length, respectively. In addition, $R$ stands for the crystal curvature and $\theta$ is the Bragg angle. As to obtain this formula, a rectangular segment was supposed. For a discrete conical crystal that is cut into small rhomboids, this formula is slightly different and can be approximated by

$$
\Omega=\frac{S_{x} S_{y}}{2}\left(\sum_{i=1}^{n_{y}} \frac{n_{0}+1-i}{R_{i}^{2}}\right) \sin ^{3} \theta,
$$

where now $S_{x}$ and $S_{y}$ are the principal axis lengths of the rhomboids, $n_{0}$ is the number of rhomboids that fit the first conical ring with radius $R_{0}$, and $n_{y}$ is the number of conical rings used. In our case, $S_{x}=1 \mathrm{~mm}, S_{y}=2 \mathrm{~mm}$, and $n_{0}=98$ rhomboids. Using these equations, a comparison between the total solid angle for this spectrometer and the one described in Ref. 10 [a $25 \mathrm{~cm}$ radius of curvature cylindrical crystal of $\mathrm{Si}(444)]$ is shown in Fig. 4 as a function of the energy of the incident photons. It is possible to see how, with small rhomboidal cuts and a specific discrete conical surface, the total solid angle is large, therefore being useful for faster acquisition time. 


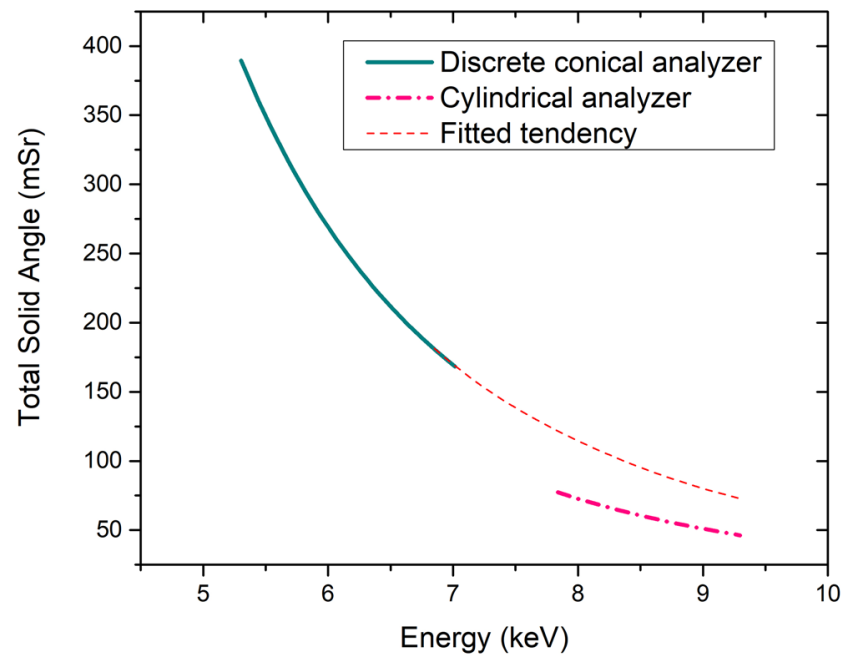

FIG. 4. Calculation of the energy dependence of total solid angle for our discrete conical crystal analyzer (green solid line) and for the cylindrical crystal analyzer proposed in Ref. 10 (pink dotted-dashed line). The fitted tendency in the proposed crystal of the total solid angle for higher energies is shown (red dashed line).

\section{FABRICATION OF THE CRYSTAL ANALYZER}

After fixing the parameters, the corresponding crystal holder was designed in Autodesk Inventor ${ }^{18}$ (Fig. 5). Due to the discreteness of the definition of the surface, some corrections were necessary to be made to have a continuous surface. The problem that arises is that when the tilt angle $\delta_{i}$ of the $i$ th conical section is introduced, a space between the sections $i$ and $(i-1)$ is evident. It is possible to fill this missing space with an extension of the corresponding conical section. This approximation will definitely introduce an error into the discrimination capability of the crystal, which scales proportionally to the size of $w$. The resulting shape is the one depicted in Fig. 5 in which 36 conical sections were used to support a crystal with a total length of $36 \mathrm{~mm}$ (see the supplementary material for the parameters of all conical sections).

An STL format file was generated from the assembly of the 36 pieces, as a unique piece. The resulting piece was fabricated with polypropylene by $3 \mathrm{D}$ printing.

The crystal was cut into small rhomboids using a $20 \mu \mathrm{m}$ thick disk to perform the cuts, following the idea of von Hámos. ${ }^{19}$ The

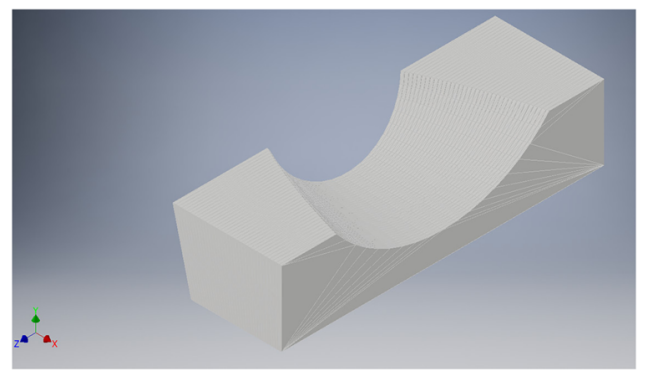

FIG. 5. Resulting shape of the crystal holder.

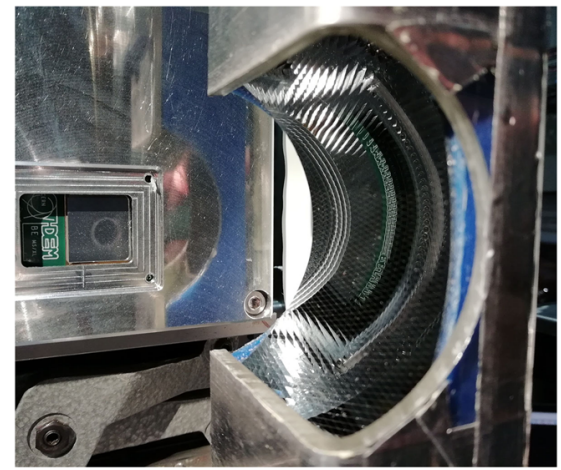

FIG. 6. Si(220) crystal shaped to its final geometry, glued onto the polypropylene substrate (blue). The cuts on the crystal form small rhomboids. The PSD can be observed behind the crystal analyzer in the left part of the image.

crystal was cut in such a way to leave a $0.1 \mathrm{~mm}$ layer intact. Afterward, the crystal was glued onto the surface of the crystal holder, with the final result shown in Fig. 6.

\section{TESTING THE SYSTEM}

The system was tested at the XRF-D09B beamline ${ }^{20}$ of the LNLS synchrotron in Campinas-SP, Brazil. The position sensitive detector used for commissioning was a WARMPix LNLS 65K-Si Detector System (v1.0), developed in-house by the Detector Group of the LNLS. This detector is composed of a Medipix module, developed by the European Organization for Nuclear Research (CERN) ${ }^{21}$ The size of this single-chip is $14 \times 14 \mathrm{~mm}^{2}$ and consists in an array of $256 \times$ 256 pixels $^{2}$ with $55 \times 55 \mu \mathrm{m}^{2}$ size per pixel. Figure 7 shows a picture of the experimental setup. As it can be seen, the sample, the crystal analyzer, and the PSD are placed on their respective positions (as described in Sec. III). A silicon drift detector (SDD) (Ketek AXASA) can also be seen on the opposite side of the PSD. This detector is intended to measure the fluorescence spectrum of a thin sample at the same time in the transmission mode.

Due to the limitation in detection area size, the positionsensitive detector was mounted on a motorized stage, allowing it to move parallel to the incident beam, thus covering the $40 \mathrm{~mm}$ region of interest. This allowed fixing the PSD at different positions along

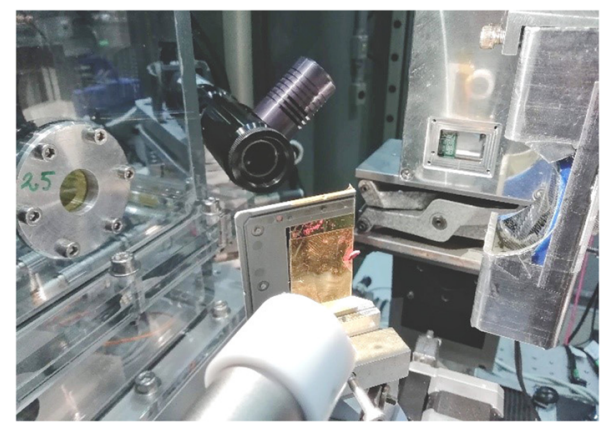

FIG. 7. Picture of the experimental setup used during the commissioning of the WDS at the LNLS XRF-D09B beamline. 
the $y$-axis so as to search for different energy peaks. The CNPEM is currently working on larger arrays that will suit well for these kinds of spectrometers.

White beam excitation was used during system calibration to maximize the photon flux. The crystal analyzer was also set on a three motor-axis that allowed fine corrections of its position. Small angular deviations of the crystal were also corrected with goniometric cradles attached to the upper part of the crystal support.

As for the incident beam, a $40 \mu \mathrm{m}$ focal spot was set at the sample position. The achievable focal spot by means of $\mathrm{KB}$ at this beamline is smaller, but this value was chosen as to have a greater incoming flux.

Once all the components were aligned, an XRF spectrum of a manganese compound $\left(\mathrm{MnO}_{2}\right)$ was measured. The resulting spectrum is shown in Fig. 8. The measured image on the detector is shown in the upper part of the plot of this figure, where it is plotted as a function of the pixel number on the $\mathrm{X}$ and $\mathrm{Y}$ directions. Two lines can be distinguished, which correspond to two different emission energies very close to each other. XRF spectra for a specific elapsed time as a function of the position along the $\mathrm{Y}$-direction (dispersive direction) were obtained integrating all counts over the detector's pixels on the X-direction [Fig. 8(b)].

Equation (6) was used to calibrate the pixel's position along the dispersive direction in energy. The result is shown in Fig. 9. From energy calibration, it is possible to clearly identify the observed peaks as the $\mathrm{Mn}-\mathrm{Ka}_{1}(5898.70 \mathrm{eV})$ and $\mathrm{Mn}-\mathrm{Ka}_{2}(5887.60 \mathrm{eV})$ emission lines. OriginPro $9^{22}$ software was used to fit the Full Width at Half Maximum (FWHM) of each peak to obtain the actual energy resolution of the system. In this case, the FWHM for the $\mathrm{Mn}-\mathrm{Ka}_{1}$ line was of only $8.25 \mathrm{eV}$. As it may be seen, $\mathrm{Ka}_{1}$ and $\mathrm{Ka}_{2}$ lines are separated only by $11.1 \mathrm{eV}$ and are being discriminated in Fig. 9.
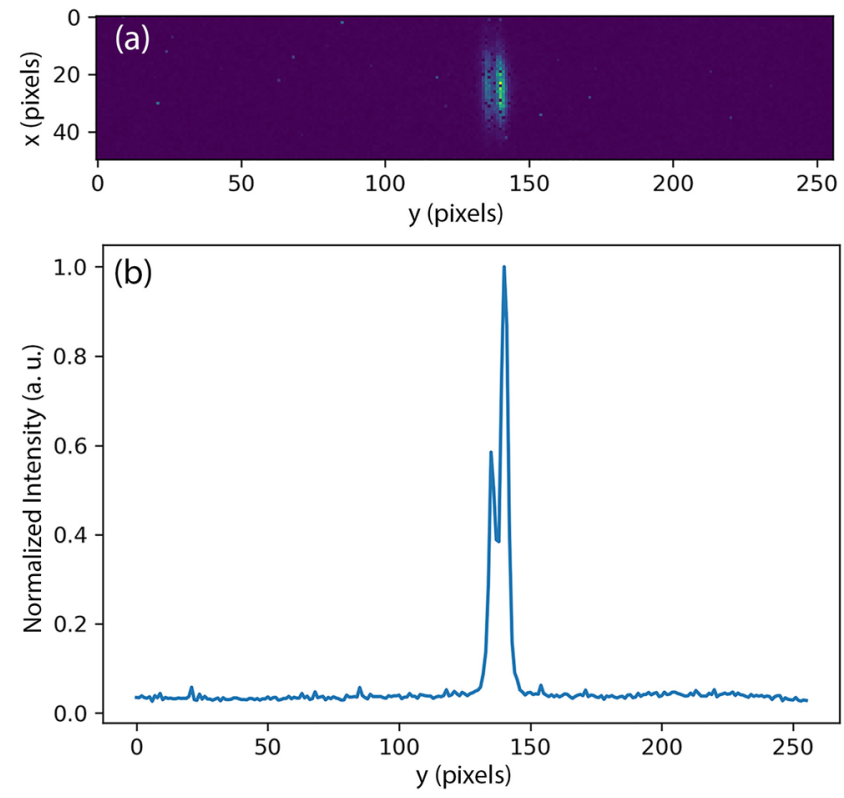

FIG. 8. (a) Image acquired by the PSD detector from a $\mathrm{MnO}_{2}$ sample (upper plot) and (b) high-resolution XRF spectrum after integration of counts over all pixels on the X-direction (lower plot).

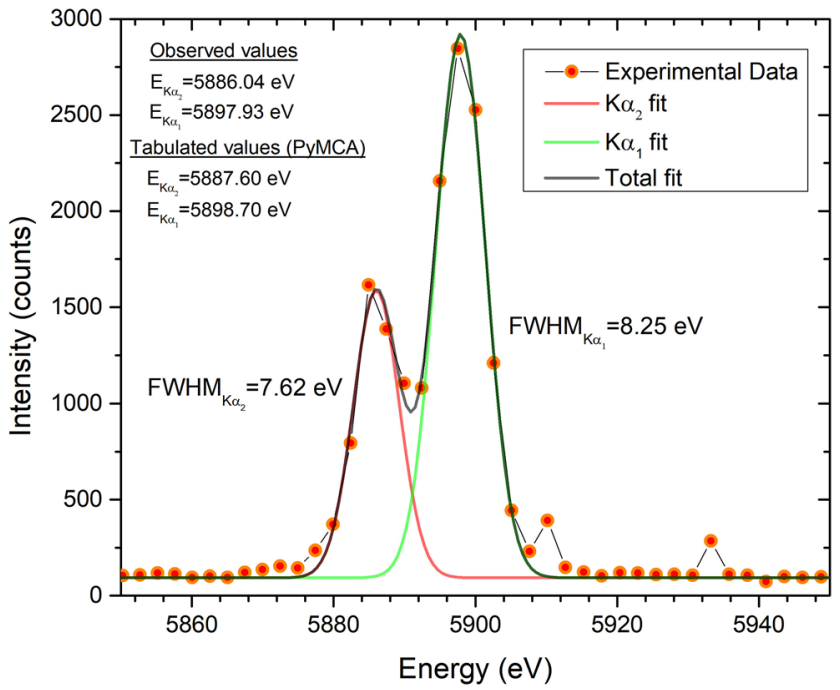

FIG. 9. K $\alpha$ region of the manganese sample's spectrum measured with the spectrometer described in this work.

From the comparison between the simulated spectrum (Fig. 3) and the measured one (Fig. 9), it is possible to see that the results agree quite well. Both spectra coincide in their main features. The relative intensity between $K \alpha_{1}$ and $K \alpha_{2}$ peaks is close to 0.5 in both cases. The largest difference encountered is the FWHM of the $K \alpha_{1}$ peak, which resulted in a ratio of full width at half maxima between the simulation and experimental result of 0.58 . Nevertheless, from the simulation, it is possible to see that imperfections in the crystal's geometry will contribute to neighboring pixels. There is an error included in the measurement that is due to the crystal cuts, as in any kind of Von Hamos spectrometer. Therefore, a difference between the measured and simulated spectra was expected. This

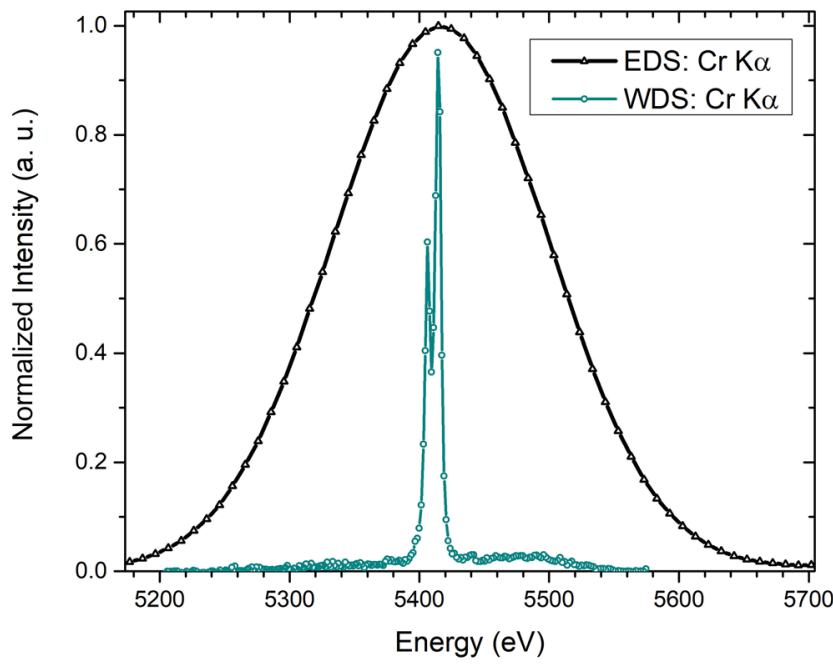

FIG. 10. Comparison between the pure $\mathrm{Cr}$ sample spectrum acquired with an SDD detector (energy-dispersive system) and the spectrometer proposed in this work. 
difference is attributed also to the gluing process in which some crystals may not lie in the correct position or with the correct orientation and also to the focalization of the incident beam (finitesize source effects). Despite this small difference, we achieved the desired energy resolution for a wide range of energies with a low-cost spectrometer.

As to test and compare the energy resolution of the discreteconical spectrometer with the SDD available in the D09B-XRF beamline, a pure chromium sample was measured with both spectrometers. The corresponding spectra are shown in Fig. 10.

It is possible to see that the SDD is not able to discriminate the fine structure in the $K \alpha$ peak as the discrete-conical analyzer does. The improvement in resolution for this energy range was of a factor of 30 (184 eV for the SDD detector compared with $6 \mathrm{eV}$ for the spectrometer) at the D09B-XRF beamline.

\section{CONCLUSION}

In this work, we successfully designed, fabricated, and commissioned a spectrometer based on a discrete-conical crystal analyzer. An energy resolution of about $8 \mathrm{eV}$ for the $M n-K \alpha_{1}$ can be achieved by using a $\mathrm{Si}(220)$ crystal, allowing to use it as a high-resolution $\mathrm{XRF}$ spectrometer in an energy range spanning from $5.3 \mathrm{keV}$ to 7.0 $\mathrm{keV}$. It is important to note that the fabricated crystal is just a prototype example. Different energy ranges may be covered by using other diffraction planes of the silicon crystal or by using other crystals as well. The spectrometer is compact with a sample-detector distance of $146 \mathrm{~mm}$. Since the crystal is only of $36 \mathrm{~mm}$ long, the total subtended solid angle is small, demanding larger collection times for data acquisition than typical SDD detectors. Nevertheless, this work shows that the spectrometer can operate as an "one-shot" WDS system with a significant improvement in energy resolution as compared with those achievable with current EDS systems.

These types of spectrometers can be considered as a very good option for high-resolution experiments at the new upcoming highbrilliance synchrotron radiation sources or at $\mathrm{x}$-ray free electron laser facilities as well. In both cases, some considerations may be needed regarding radiation damage for the first order interactions such as fluorescence, but a spectrometer such as the one presented in this work may be very well suited to study the second order interactions, such as RIXS, which have very low probability of occurrence and thus are very photon hungry. It may also be used in a conventional laboratory source, but acquisition times may be very long in such cases. Experiments under low-vacuum environment conditions may improve the counting rate in such laboratories in order to reduce the absorption in air.

\section{SUPPLEMENTARY MATERIAL}

See the supplementary material for the complete table of values of the parameters for each of the 36 conical sections used in the $\mathrm{Si}(220)$ crystal analyzer prototype.

\section{ACKNOWLEDGMENTS}

The authors would like to thank the CONICET and the CNPEM for the funding that allowed the fabrication of the spectrometer as well as the measurements of this work through several experimental proposals. A special thanks to M. Honnicke, from the UNILA, who supplied the Si crystal wafer for this project, and to H. R. Ribeiro and J. L. da Silva for their contributions in the design and fabrication process of the crystal analyzer. The authors would like to thank the LNLS detector group, particularly to D. de Paiva Magalhães, for the support on the PSD configuration.

\section{REFERENCES}

${ }^{1}$ J. Szlachetko, M. Cotte, J. Morse, M. Salomé, P. Jagodzinski, J.-Cl. Dousse, J. Hoszowska, Y. Kayser, and J. Susini, "Wavelength-dispersive spectrometer for $\mathrm{X}$-ray microfluorescence analysis at the X-ray microscopy beamline ID21 (ESRF)," J. Synchrotron Radiat. 17, 400-408 (2010).

${ }^{2}$ M. Torres Deluigi, F. M. F. de Groot, G. López-Díaz, G. Tirao, G. Stutz, and J. Riveros de la Vega, "Core and valence structures in $\mathrm{k} \beta$ x-ray emission spectra of chromium materials," J. Phys. Chem. C 118, 22202-22210 (2014).

${ }^{3}$ P. A. Lee, P. H. Citrin, P. Eisenberger, and B. M. Kincaid, "Extended x-ray absorption fine structure-its strengths and limitations as a structural tool," Rev. Mod. Phys. 53, 769-806 (1981).

${ }^{4}$ A. Kotani and S. Shin, "Resonant inelastic x-ray scattering spectra for electrons in solids," Rev. Mod. Phys. 73, 203 (2001).

${ }^{5}$ D. Kirsanov, V. Panchuk, A. Goydenko, M. Khaydukova, V. Semenov, and A. Legin, "Improving precision of X-ray fluorescence analysis of lanthanide mixtures using partial least squares regression," Spectrochim. Acta Part B 113, 126-131 (2015)

${ }^{6}$ D. S. Zinin, N. N. Bushuev, and V. V. Kuznetsov, "X-ray fluorescence determination of $\mathrm{La}, \mathrm{Ce}, \mathrm{Pr}, \mathrm{Nd}$, and $\mathrm{Sm}$ in industrial sediments of calcium sulfate using linear regression analysis," J. Anal. Chem. 72, 279-288 (2017).

${ }^{7}$ H. Ebel, M. Mantler, N. Gurker, and J. Wernisch, "An x-ray spectrometer with a position-sensitive wire detector (PSD)," X-Ray Spectrom. 12, 47-49 (1983).

${ }^{8}$ G. Tirao, C. Cusatis, and G. Stutz, "Construction of a focusing high-resolution crystal analyser for X-rays,” X-Ray Spectrom. 31, 158-161 (2002).

${ }^{9}$ K. Hayashi, K. Nakajima, K. Fujiwara, and S. Nishikata, "Wave-dispersive $\mathrm{X}$-ray spectrometer for simultaneous acquisition of several characteristic lines based on strongly and accurately shaped Ge crystal," Rev. Sci. Instrum. 79, 033110 (2008).

${ }^{10}$ J. Szlachetko, M. Nachtegaal, E. de Boni, M. Willimann, O. Safonova, J. Sa, G. Smolentsev, M. Szlachetko, J. A. van Bokhoven, J.-Cl. Dousse, J. Hoszowska, Y. Kayser, P. Jagodzinski, A. Bergamaschi, B. Schmitt, C. David, A. Lücke, and A. von Hamos, "X-ray spectrometer based on a segmented-type diffraction crystal for single-shot $\mathrm{x}$-ray emission spectroscopy and time-resolved resonant inelastic X-ray scattering studies," Rev. Sci. Instrum. 83, 103105 (2012).

${ }^{11}$ J. Szlachetko, J.-Cl. Dousse, J. Hoszowska, M. Pajek, R. Barrett, M. Berset, K. Fennane, A. Kubala-Kukus, and M. Szlachetko, "High-resolution study of $\mathrm{X}$-ray resonant Raman scattering at the K edge of silicon," Phys. Rev. Lett. 97, 073001 (2006).

${ }^{12}$ K. Morishita, K. Hayashi, and K. Nakajima, "One-shot spectrometer for several elements using an integrated conical crystal analyzer," Rev. Sci. Instrum. 83, 013112 (2012).

${ }^{13}$ K. Nakajima, K. Fujiwara, W. Pan, and H. Okuda, "Shaped silicon-crystal wafers obtained by plastic deformation and their application to silicon-crystal lenses," Nat. Mater. 4, 47-50 (2005).

${ }^{14}$ J. I. Robledo, Avances en Espectroscopía por Dispersión Inelástica Resonante de Rayos X en Baja y Alta Resolución (Universidad Nacional de Córdoba, 2019).

${ }^{15}$ MATLAB, The Mathworks Inc., 2010.

${ }^{16}$ E. Bergbäck Knudsen, A. Prodi, J. Baltser, M. Thomsen, P. Kjær Willendrup, M. Sanchez del Rio, C. Ferrero, E. Farhi, K. Haldrup, A. Vickery, R. Feidenhans'l, K. Mortensen, M. Meedom Nielsen, H. Friis Poulsen, S. Schmidt, and K. Lefmann, 
"McXtrace: A Monte Carlo software package for simulating X-ray optics, beamlines and experiments," J. Appl. Crystallogr. 46, 679-696 (2013).

${ }^{17}$ V. A. Solé, E. Papillon, M. Cotte, Ph. Walter, and J. Susini, "A multiplatform code for the analysis of energy-dispersive X-ray fluorescence spectra," Spectrochim. Acta Part B 62, 63-68 (2007).

${ }^{18}$ Autodesk Inventor, Autodesk Inventor Tooling, Autodesk ${ }^{\circledR}$, San Rafael, California, USA, 2009

${ }^{19} \mathrm{~L}$. von Hámos, "Formation of true X-ray images by reflection on crystal mirrors," Z. Kristallogr. - Cryst. Mater. 101(1) , 17-29 (1939).
${ }^{20}$ C. A. Pérez, M. Radtke, H. J. Sánchez, H. Tolentino, R. T. Neuenshwander, W. Barg, M. Rubio, M. I. S. Bueno, I. M. Raimundo, and J. J. R. Rohwedder, "Synchrotron radiation X-ray fluorescence at the LNLS: Beamline instrumentation and experiments," X-Ray Spectrom. 28, 320-326 (1999).

${ }^{21}$ R. Ballabriga, J. Alozy, G. Blaj, M. Campbell, M. Fiederle, E. Frojdh, E. H. M. Heijne, X. Llopart, M. Pichotka, S. Procz, L. Tlustos, and W. Wong, "The Medipix3RX: A high resolution, zero dead-time pixel detector readout chip allowing spectroscopic imaging," J. Instrum. 8, C02016 (2013).

${ }^{22}$ OriginLab, Origin Pro 9, Northhampton, MA, 2003. 